GUIMARÃES, T.G.; FONTES, P.C.R.; PEREIRA, P.R.G.; ALVAREZ V., V.H.; MONNERAT, P.H. Determinação dos teores de nitrogênio na seiva do tomateiro por meio de medidor portátil. Horticultura Brasileira, Brasília, v. 16, n. 2, p. 144-151, novembro de 1998.

\title{
Determinação dos teores de nitrogênio na seiva do tomateiro por meio de medidor portátil. ${ }^{1}$
}

\author{
Tadeu G. Guimarães ${ }^{2}$; Paulo C. R. Fontes ${ }^{3}$; Paulo R. G. Pereira ${ }^{4}$; Victor H. Alvarez V.; Pedro H. \\ Monnerat ${ }^{6}$. \\ 2, 3 e 4 UFV - Departamento de Fitotecnia, 36571-000 Viçosa - MG; ${ }^{5}$ UFV - Departamento de Solos, 36571-000 Viçosa - MG; ${ }^{6}$ UENF - \\ Laboratório de Fitotecnia - CCTA, 28015-620 Campos - RJ.
}

\section{RESUMO}

O presente estudo objetivou determinar teores e níveis críticos de nitrogênio na forma de nitrato $\left(\mathrm{N}-\mathrm{NO}_{3}\right)$ na seiva do pecíolo de tomateiros utilizando-se medidor portátil, e estabelecer a relação destes valores com os teores de $\mathrm{N}-\mathrm{NO}_{3}$ na matéria seca dos pecíolos, e de N-orgânico e de $\mathrm{N}$-total na matéria seca do limbo. Plantas de tomateiro (Lycopersicon esculentum), cv. Santa Clara, cultivadas em vasos contendo amostras de dois solos (argiloso $=\mathrm{Vi}$; areia-franca $=$ $\mathrm{TM})$, foram submetidas a cinco doses de $\mathrm{N}(25,125,225,325$ e 425 $\mathrm{mg} / \mathrm{dm}^{3}$ ) aplicadas parceladamente, via solução nutritiva. As folhas opostas ao $1^{\circ} \mathrm{e}$ ao $3^{\circ}$ cacho foram amostradas por ocasião do início da antese das flores do respectivo cacho, e as concentrações de N$\mathrm{NO}_{3}$ na seiva dos pecíolos foram determinadas utilizando medidor portátil equipado com microeletrodo seletivo ao $\mathrm{N}-\mathrm{NO}_{3}$. Determinou-se, também, as concentrações de $\mathrm{N}^{-\mathrm{NO}_{3}}$ na matéria seca do pecíolo e do limbo, e as concentrações de N-orgânico na matéria seca do limbo, ambas por colorimetria. Os valores de $\mathrm{N}$-total na matéria seca do limbo foram obtidos a partir da soma dos teores de $\mathrm{N}-\mathrm{NO}_{3}$ e de $\mathrm{N}$-orgânico. Os níveis críticos das formas de $\mathrm{N}$ foram maiores no solo TM, e em ambos os solos, maiores na folha oposta ao $1^{\circ}$ cacho. Os níveis críticos de $\mathrm{N}-\mathrm{NO}_{3}$ nas seiva das folhas opostas ao $1^{\circ} \mathrm{e}$ ao $3^{\circ}$ cacho foram, respectivamente 2.581 e $1.085 \mathrm{mg} / \mathrm{L}$ para o solo Vi, e 2.616 e $1.690 \mathrm{mg} / \mathrm{L}$ para o solo TM. Os teores de $\mathrm{N}-\mathrm{NO}_{3}$ na matéria seca dos pecíolos, e de $\mathrm{N}$-orgânico e de $\mathrm{N}$-total na matéria seca do limbo foram estimados com relativa precisão à partir dos teores de $\mathrm{N}^{-\mathrm{NO}_{3}}$ na seiva, em ambas as amostragens feitas nas plantas cultivadas no solo TM, e nas folhas opostas ao $3^{\circ}$ cacho das plantas cultivadas no solo Vi.

Palavras-chave: Lycopersicon esculentum, nitrato, nivel crítico.

\begin{abstract} Deter.

The aim of this study was to determine concentrations and critical levels of $\mathrm{NO}_{3}-\mathrm{N}$ in tomato petiole sap by a quick test using a portable meter, and the relationship between these values and those of $\mathrm{NO}_{3}$ $\mathrm{N}$ on petiole dry matter, and of organic and total $\mathrm{N}$ on leaf blade dry matter. Tomato (Lycopersicon esculentum Mill.) plants, cv. Santa Clara, grown in pots of two soil samples (clay $=\mathrm{Vi}$ and sand-course $=\mathrm{TM})$, were submitted to five $\mathrm{N}$ rates $(25,125,225,325$ and 425 $\mathrm{mg} / \mathrm{dm}^{3}$ ) sidedress applied as nutrient solutions. Leaves opposite to $1^{\text {st }}$ and $3^{\text {rd }}$ clusters were sampled at the onset of anthesis of each cluster, and petiole sap $\mathrm{NO}_{3}-\mathrm{N}$ concentrations were determined with a portable meter equipped with a microelectrode selective to $\mathrm{NO}_{3}$ $\mathrm{N}$. Additionally, organic $\mathrm{N}$ (org- $\mathrm{N}$ ) and $\mathrm{NO}_{3}-\mathrm{N}$ were determined in leaf blade dry matter, and $\mathrm{NO}_{3}-\mathrm{N}$ was determined in petiole dry matter. Total-N (tot-N) concentrations in blade dry matter were the sum of $\mathrm{NO}_{3}-\mathrm{N}$ and organic-N. Critical $\mathrm{N}$ levels were highest on the TM soil, and for both soils, highest on the $1^{\text {st }}$ cluster. $\mathrm{NO}_{3}-\mathrm{N}$ critical levels in petiole sap of leaves opposite to $1^{\text {st }}$ and $3^{\text {rd }}$ clusters were 2,581 and 1,085 $\mathrm{mg} / \mathrm{L}$ on soil $\mathrm{Vi}$, and 2,616 and $1,690 \mathrm{mg} / \mathrm{L}$ on soil $\mathrm{TM}$, respectively. Concentrations of $\mathrm{NO}_{3}-\mathrm{N}$ in petiole dry matter and of org- $\mathrm{N}$ and tot- $\mathrm{N}$ in blade dry matter were well estimated by sap $\mathrm{NO}_{3}-\mathrm{N}$ concentrations in leaf samples taken from plants grown on soil TM, and in leaves opposite to the $3^{\text {rd }}$ cluster from plants grown on soil Vi.
\end{abstract}

Keywords: Lycopersicon esculentum, critical level, nitrate.

\section{(Aceito para publicação em 01 de outubro de 1998)}

$\mathrm{R}$ ecentemente, o manejo correto da fertilização nitrogenada do tomateiro tem sido mais enfaticamente pesquisado, visto que além de proporcionar aumentos qualitativos e quantitativos na produção, concorre para a redução dos custos de produção e da contaminação do ambiente. Dentre as principais medidas apropriadas para o manejo da fertilização nitrogenada destacam-se as aplicações parceladas e localizadas dos fertilizantes, procurando-se quantificar as doses a partir dos resultados das análises de solo e da planta.

As análises de solo têm sido utilizadas em outros países para a recomendação de adubação nitrogenada para diversas culturas como milho (Binford et al., 1992; Blackmer et al., 1993), batata (Neeteson et al., 1986; Neeteson, 1989; Neeteson \& Swetsloot, 1989; MacKerron et al., 1993), beterraba açucareira
(Neeteson, 1989; Neeteson \& Swetsloot, 1989), dentre outras. Entretanto, a ausência de métodos de análise de $\mathrm{N}$ no solo que estimem, confiavelmente, as quantidades necessárias à adequada nutrição do tomateiro faz com que o seu estado nutricional e possíveis recomendações de adubação nitrogenada sejam feitas empiricamente ou com base em determinações das concentrações de N-total e N$\mathrm{NO}_{3}$ nos tecidos foliares (Coltman, 1988).

\footnotetext{
${ }^{1}$ Parte da tese de Doutorado do primeiro autor; ${ }^{2}$ Endereço atual: UFU - DEAGO, Campus Umuarama, 38400-902 Uberlândia - MG; ${ }^{3}$ 5
} Bolsistas do CNPq. 
Uma vez que o $\mathrm{N}_{-} \mathrm{NO}_{3}$ é a forma de $\mathrm{N}$ preferencialmente absorvida e translocada pelo tomateiro, a sua concentração nas folhas tem sido mais indicada para a verificação do "status" de N e recomendação de adubação nitrogenada (Coltman, 1988). Entretanto, as determinações de $\mathrm{N}-\mathrm{NO}_{3}$ na matéria seca têm custo elevado, e gasta-se muito tempo entre a tomada da amostra e a obtenção dos resultados (Minnoti et al., 1989). Assim, a utilização de testes rápidos em campo para a avaliação do estado nutricional do tomateiro em $\mathrm{N}$ é uma alternativa promissora para a exploração racional da cultura.

Sob condições de cultivos comerciais, sejam em ambientes protegidos ou no campo, a análise da seiva dos pecíolos do tomateiro é um dos métodos mais rápidos e, de certa forma, mais confiáveis, para se verificar o "status" em N das culturas (Coltman, 1987; Sarro et al., 1985 e 1987). A análise da seiva informa o estado nutricional da planta em um determinado instante, com imediata aplicação no manejo da fertilização nitrogenada (Sarro et al., 1985 e 1987). As determinações das concentrações de $\mathrm{NO}_{3}$ na seiva podem ser feitas utilizando-se eletrodos ou tiras de papel específicos (Coltman, 1988). Assim, a análise de $\mathrm{NO}_{3}$ na seiva do pecíolo, a qual mede o balanço entre a demanda e o suprimento de $\mathrm{N}$ pelo solo, poderá ser indicado como critério para a realização de diagnóstico do "status" nitrogenado do tomateiro de maneira fácil e rápida.

O presente estudo objetivou determinar, utilizando microeletrodo seletivo, os níveis críticos de $\mathrm{N}-\mathrm{NO}_{3}$ na seiva do pecíolo, de $\mathrm{N}_{-} \mathrm{NO}_{3}$ na matéria seca de pecíolos, e de N-orgânico e de N-total na matéria seca do limbo de folhas do tomateiro, como também estudar a relação existente entre os valores das concentrações de $\mathrm{N}-\mathrm{NO}_{3}$, N-orgânico e $\mathrm{N}$-total na matéria seca e os das concentrações de $\mathrm{N}_{-} \mathrm{NO}_{3}$ na seiva.

\section{MATERIAL E MÉTODOS}

O experimento foi conduzido em casa-de-vegetação do Departamento de Fitotecnia da Universidade Federal de Viçosa (DFT-UFV), no período de 24 de novembro de 1995 a 27 de janeiro de

Tabela 1. Procedência e principais características físicas e químicas das amostras de solo utilizadas. Viçosa, Universidade Federal de Viçosa, 1995/1996.

\begin{tabular}{|c|c|c|}
\hline Características & Amostra (Vi) & Amostra (TM) \\
\hline Procedência & Viçosa-MG & Três Marias-MG \\
\hline Argila (dag/kg) & 44 & 12 \\
\hline Classificação Textural & Argiloso & Areia-Franca \\
\hline Densidade Aparente $\left(\mathrm{g} / \mathrm{cm}^{3}\right)$ & 1,095 & 1,360 \\
\hline Capacidade de Campo (kg/kg) & 0,329 & 0,065 \\
\hline Matéria Orgânica (dag/kg) & 1,58 & 0,46 \\
\hline $\mathrm{pH}\left(\mathrm{H}_{2} \mathrm{O}\right)$ & 5,5 & 4,7 \\
\hline $\mathrm{P}\left(\mathrm{mg} / \mathrm{dm}^{3}\right)$ & 81,1 & 2,1 \\
\hline $\mathrm{K}\left(\mathrm{mg} / \mathrm{dm}^{3}\right)$ & 71 & 16 \\
\hline $\mathrm{Al}^{\beta+}\left(\mathrm{cmol}_{\mathrm{c}} / \mathrm{dm}^{3}\right)$ & 0,0 & 0,3 \\
\hline $\mathrm{Ca}^{2+}\left(\mathrm{cmol}_{\mathrm{c}} / \mathrm{dm}^{3}\right)$ & 3,2 & 0,1 \\
\hline $\mathrm{Mg}^{2+}\left(\mathrm{cmol}_{\mathrm{c}} / \mathrm{dm}^{3}\right)$ & 0,7 & 0,0 \\
\hline $\mathrm{H}+\mathrm{Al}\left(\mathrm{cmol}_{\mathrm{c}} / \mathrm{dm}^{3}\right)$ & 2,7 & 3,6 \\
\hline $\mathrm{SB}\left(\mathrm{cmol}_{\mathrm{c}} / \mathrm{dm}^{3}\right)$ & 4,08 & 0,14 \\
\hline CTC t $\left(\mathrm{cmol}_{\mathrm{c}} / \mathrm{dm}^{3}\right)$ & 4,08 & 0,44 \\
\hline CTC T $\left(\mathrm{cmol}_{\mathrm{c}} / \mathrm{dm}^{3}\right)$ & 6,78 & 3,74 \\
\hline $\mathrm{V}(\%)$ & 60,2 & 3,7 \\
\hline $\mathrm{m}(\%)$ & 0,0 & 68,2 \\
\hline
\end{tabular}

1996, utilizando-se vasos de 12,0 L com $10 \mathrm{dm}^{3}$ de amostras de dois solos de texturas e condições de fertilidade contrastantes, cujas principais características químicas e físicas encontram-se na Tabela 1. A amostra de solo de Viçosa-MG (Vi), de textura argilosa, é proveniente de uma área destinada ao cultivo de hortaliças na Universidade Federal de Viçosa, e que seria utilizada para a condução de ensaios de campo, envolvendo estudos sobre fertilização nitrogenada do tomateiro, enquanto que a amostra de solo de Três Marias-MG (TM) de textura areia-franca, é proveniente de área sem exploração agrícola atual.

No dia da semeadura (24/11/95) foi feito a calagem no solo TM aplicandose uma mistura de $\mathrm{CaCO}_{3}$ e $\mathrm{MgO}$ (4:1 mols), ambos p.a., para elevar a saturação de bases a $60 \%$, e $300 \mathrm{mg} / \mathrm{dm}^{3}$ de P na forma de superfosfato triplo; no solo argiloso aplicou-se $500 \mathrm{mg} / \mathrm{dm}^{3}$ de P utilizando-se a mesma fonte. Metade da dose de P foi aplicada em $1 \mathrm{dm}^{3}$ do volume do solo, o qual foi localizado superficialmente nos vasos, e a outra metade aplicada nos $9 \mathrm{dm}^{3}$ restantes.

Em seguida, os vasos foram irrigados e seis sementes de tomate de cultivar Santa Clara foram semeadas. Após a emergência, foi feito desbaste, deixando-se uma planta por vaso.

Os tratamentos constaram de cinco doses de $\mathrm{N}(25,125,225,325$ e $425 \mathrm{mg} /$ $\mathrm{dm}^{3}$ ), parceladas em seis aplicações em cobertura, via solução nutritiva $(25 \%$ como $\mathrm{N}-\mathrm{NH}_{4}^{+}$), utilizando $\mathrm{NH}_{4} \mathrm{NO}_{3}$, $\mathrm{KNO}_{3}$ e $\mathrm{NaNO}_{3}$, todas p.a., como fontes. As doses de $\mathrm{N}$ foram parceladas obedecendo o seguinte cronograma, em dias após a semeadura (DAS): 5\% em cada aplicação aos 14 e 24 DAS; $10 \%$ aos 33 DAS; $20 \%$ aos 43 DAS; $30 \%$ aos 49 DAS, e $30 \%$ aos 54 DAS. As unidades experimentais foram dispostas na estufa seguindo o delineamento de blocos casualizados, com 5 repetições.

Foram aplicados $\mathrm{K}, \mathrm{Ca}, \mathrm{Mg}, \mathrm{S}$ e micronutrientes em cobertura, na forma de soluções nutritivas, sendo os micronutrientes também aplicados por via foliar. As aplicações de K e S foram realizadas conjuntamente com as adubações nitrogenadas, seguindo o mesmo parcelamento destas, totalizando $182 \mathrm{e}$ $40 \mathrm{mg} / \mathrm{dm}^{3}$ de $\mathrm{K}$ e de $\mathrm{S}$, respectivamente. No solo TM aplicou-se 0,975 e 0,45 $\mathrm{cmol} / \mathrm{dm}^{3}$ de $\mathrm{Ca}$ e de $\mathrm{Mg}$, respectivamente, enquanto que no solo $\mathrm{Vi}$ aplicouse 0,75 e $0,3 \mathrm{cmol}_{\mathrm{c}} / \mathrm{dm}^{3}$ de $\mathrm{Ca}$ e de $\mathrm{Mg}$, 
respectivamente, em cobertura via soluções nutritivas.

No solo Vi as doses de micronutrientes totalizaram $2 \mathrm{mg} / \mathrm{dm}^{3}$ de $\mathrm{Zn} ; 1$ $\mathrm{mg} / \mathrm{dm}^{3}$ de $\mathrm{B} ; 1 \mathrm{mg} / \mathrm{dm}^{3}$ de $\mathrm{Cu} ; 0,5 \mathrm{mg} /$ $\mathrm{dm}^{3}$ de $\mathrm{Mn}$, e $0,2 \mathrm{mg} / \mathrm{dm}^{3}$ de $\mathrm{Mo}$, enquanto que no solo TM estas totalizaram $4 \mathrm{mg} / \mathrm{dm}^{3}$ de $\mathrm{Zn} ; 1,5 \mathrm{mg} / \mathrm{dm}^{3}$ de B; 1,5 $\mathrm{mg} / \mathrm{dm}^{3}$ de $\mathrm{Cu} ; 1 \mathrm{mg} / \mathrm{dm}^{3}$ de $\mathrm{Mn}$, e 0,4 $\mathrm{mg} / \mathrm{dm}^{3}$ de Mo. Foram feitas aplicações foliares de soluções de $\mathrm{H}_{3} \mathrm{BO}_{3}$ a 0,15 $\mathrm{dag} / \mathrm{L}, \mathrm{ZnSO}_{4} .7 \mathrm{H}_{2} \mathrm{O}$ a $0,75 \mathrm{dag} / \mathrm{L}$ e $\mathrm{Na}_{2} \mathrm{MoO}_{4} \cdot 2 \mathrm{H}_{2} \mathrm{O}$ a $0,0125 \mathrm{dag} / \mathrm{L}$ por ocasião do aparecimento do $1^{\circ}$ cacho (aos 34 DAS) e aos 55 DAS.

Pulverizações com fungicida, inseticida e acaricida foram feitas sempre que necessárias.

Por ocasião da abertura das flores do $1^{\circ}$ e do $3^{\circ}$ cachos (42 e 57 DAS, respectivamente), as folhas opostas a cada cacho foram destacadas entre as 7 e $8 \mathrm{~h}$ da manhã, acondicionadas em sacos plásticos e levadas ao laboratório, onde foram colocadas sob refrigeração. Logo em seguida, seções de $1 \mathrm{~cm}$ de comprimento foram retiradas da base dos pecíolos e maceradas, em "espremedor de alho", para extração da seiva. Com o auxílio de uma micropipeta a seiva foi coletada e analisada em medidor portátil de nitrato (C-141 Cardy Nitrate Meter- HORIBA, INC.) equipado com microeletrodo seletivo ao nitrato. Posteriormente, os pecíolos foram separados do limbo foliar, sendo ambos os materiais, separadamente, acondicionados em sacos de papel, secos em estufa de circulação forçada de ar a $75^{\circ} \mathrm{C}$ por $72 \mathrm{~h}$, pesados, moídos em moinho tipo Wiley com peneira de 20 mesh e armazenados. Nas matérias secas do pecíolo e do limbo, após a extração com água em banho-maria a $45^{\circ} \mathrm{C}$ durante $1 \mathrm{~h}$, foram determinadas as concentrações de $\mathrm{N}-\mathrm{NO}_{3}$ por colorimetria, em espectrofotômetro a $410 \mathrm{~nm}$ (Cataldo et al.,1975). Foram determinados, ainda, após digestão sulfúrica, os teores de $\mathrm{N}$ orgânico (N-org.) na matéria seca do limbo por meio do reagente de Nessler (Jackson, 1982), calculando-se, posteriormente, os valores de $\mathrm{N}$-total (N-tot) à partir da soma dos teores de $\mathrm{N}-\mathrm{NO}_{3} \mathrm{e}$ de N-orgânico.

As plantas tiveram seus pontos de crescimento eliminados imediatamente abaixo da $4^{\mathrm{a}}$ inflorescência, por ocasião

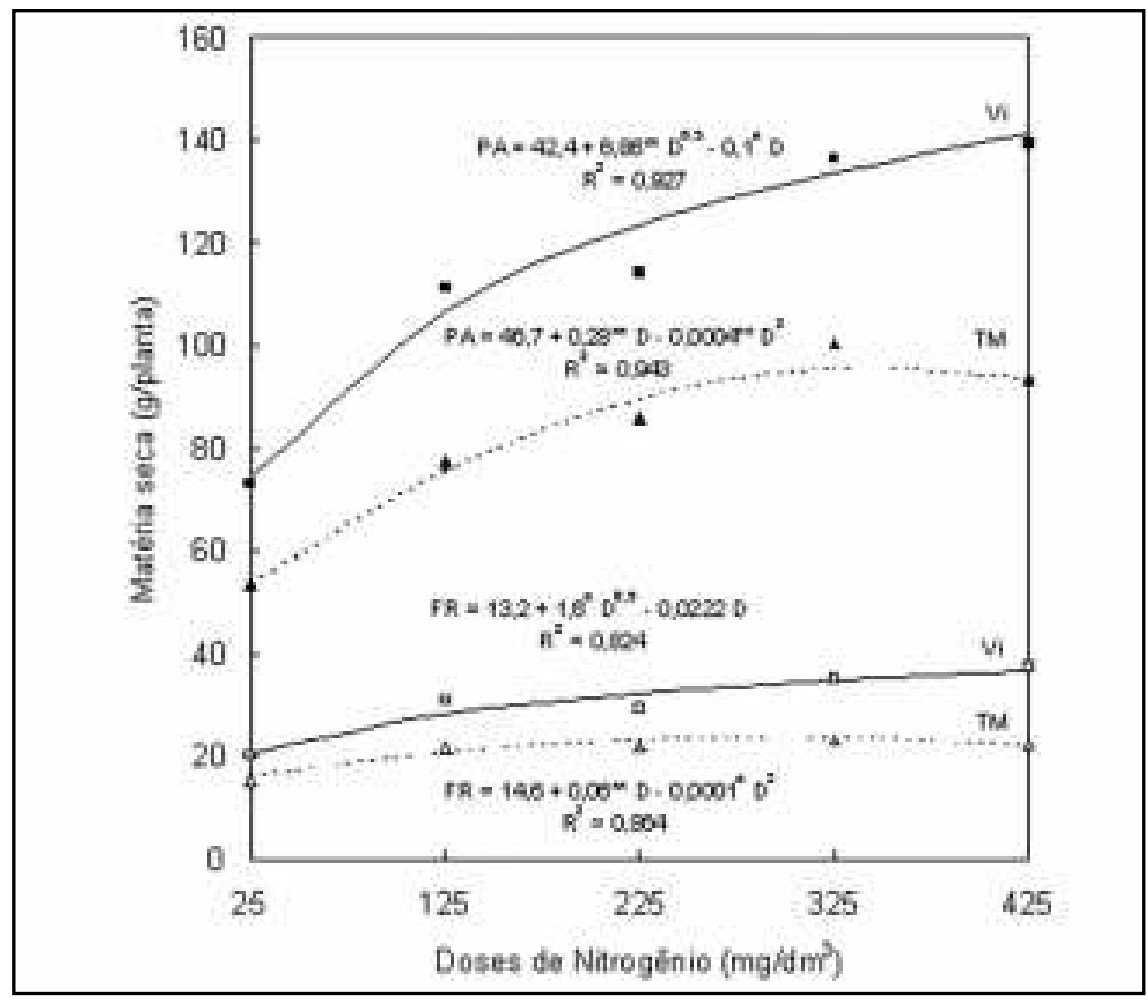

Figura 1. Produção de matéria seca de frutos $(\mathrm{FR})$ e da parte aérea (PA) do tomateiro, em solos de Viçosa (Vi) e de Três Marias (TM), em função de doses de nitrogênio. Viçosa, Universidade Federal de Viçosa, 1995/1996. do surgimento destas. Aos 64 DAS foram cortadas rente ao solo, divididas em folhas, caules e frutos, e secas em estufa de circulação forçada de ar por $72 \mathrm{~h}$ a $70{ }^{\circ} \mathrm{C}$ para determinação das produções de matéria seca.

Os dados foram submetidos às análises de variância e de correlação linear de Pearson, relacionando-se as formas de nitrogênio analisadas à produção de matéria seca da parte aérea. Posteriormente, foram ajustadas equações de regressão relacionando as variáveis dependentes às doses aplicadas de nitrogênio. Os coeficientes de correlação e os coeficientes de determinação foram testados a $1\left({ }^{* *}\right), 5\left(^{*}\right)$ e $10 \%\left(^{\circ}\right)$ de significância. A partir das doses de $\mathrm{N}$ necessárias para obtenção de $99 \%$ da produção máxima de matéria seca da parte aérea, foram calculados os níveis críticos de $\mathrm{N}_{-} \mathrm{NO}_{3}$ na matéria seca e na seiva dos pecíolos, bem como os níveis críticos de N-org e N-tot na matéria seca dos limbos foliares. Também foram ajustadas equações de regressão tomando-se os teores de $\mathrm{N}_{-} \mathrm{NO}_{3}$ na matéria seca dos pecíolos e os de $\mathrm{N}$-orgânico e $\mathrm{N}$-total na matéria seca do limbo como as variáveis dependentes, e os teores de $\mathrm{N}-\mathrm{NO}_{3}$ na seiva dos pecíolos como a variável independente.

\section{RESULTADOS E DISCUSSÃO}

No solo de Viçosa (Vi), os maiores valores das produções de matéria seca de frutos e da parte aérea foram $36,8 \mathrm{e}$ $141,3 \mathrm{~g} /$ planta, respectivamente, obtidos com a maior dose estudada, $425 \mathrm{mg} / \mathrm{dm}^{3}$ de N (Figura 1). Tal fato indica que nessa amostra de solo, para maximizar o crescimento, deveria ter sido aplicado dose de $\mathrm{N}$ ainda maior, ou ter aplicado maior percentagem das doses nas adubações iniciais. No solo de Três Marias (TM) os valores estimados máximos foram 23,4 e 95,7 g/vaso para as matérias secas de frutos e da parte aérea, respectivamente, valores obtidos com as doses de 300 e $350 \mathrm{mg} / \mathrm{dm}^{3}$ de $\mathrm{N}$, respectivamente (Figura 1). As doses responsáveis por $99 \%$ da máxima produção de matéria seca da parte aérea foram 384 e $301 \mathrm{mg} / \mathrm{dm}^{3}$ de $\mathrm{N}$, para os solos Vi e TM, respectivamente.

A produção de matéria seca da parte aérea das plantas cultivadas no solo 
Vi correlacionou-se positivamente com todas as formas de $\mathrm{N}$ analisadas, sendo que, em ambas as amostragens, as determinações dos teores de $\mathrm{N}-\mathrm{NO}_{3}$ na seiva apresentaram os maiores coeficientes de correlação (Tabela 2). No solo TM, a matéria seca da parte aérea também correlacionou-se positivamente com todas as formas de $\mathrm{N}$ analisadas (Tabela 2). Nesse solo, as correlações obtidas para os teores de $\mathrm{N}-\mathrm{NO}_{3}$ determinados na seiva, foram maiores que as obtidas quando o $\mathrm{N}_{-} \mathrm{NO}_{3}$ foi analisado na matéria seca dos pecíolos (Tabela 2).

Nas plantas cultivadas no solo Vi, os teores de $\mathrm{N}_{-} \mathrm{NO}_{3}$ na matéria seca e na seiva dos pecíolos e os teores de $\mathrm{N}$-org e de N-tot nos limbos das folhas opostas ao $1^{\circ}$ cacho associaram-se às doses de nitrogênio, podendo ser representadas por modelos dos tipos raizquadrático e quadrático, respectivamente, com coeficientes de determinação $\left(\mathrm{R}^{2}\right)$ muito baixos (Figuras 2, 3, 4 e 5, e Tabela 3). Apesar disto, os coeficientes das equações apresentaram elevada significância, indicando efeitos significativos das doses crescentes aplicadas. Tal fato deve ter ocorrido em decorrência das pequenas quantidades de $\mathrm{N}$ aplicadas $(20 \%$ do total das doses) até a ocasião da amostragem destas folhas, sendo mais um indicativo de que o limite superior do intervalo experimental para este solo, de textura argilosa, deveria ter sido maior.

Nas plantas cultivadas no solo TM, os teores de $\mathrm{N}-\mathrm{NO}_{3}$ na matéria seca e na seiva dos pecíolos das folhas opostas ao $1^{\circ}$ cacho aumentaram linearmente (Figuras 2 e 3; Tabela 3), enquanto que os teores de $\mathrm{N}$-org e N-tot no limbo destas apresentaram comportamento quadrático, com o aumento das doses de N (Figuras 4 e 5; Tabela 3). Provavelmente, a absorção de $\mathrm{NO}_{3}$ ultrapassou a demanda metabólica da planta.

Nos pecíolos das folhas opostas ao $3^{\circ}$ cacho, as concentrações de $\mathrm{N}-\mathrm{NO}_{3}$ na matéria seca e na seiva aumentaram linearmente com os aumentos nas doses de $\mathrm{N}$, em ambos os solos, porém, sendo menores que aquelas observadas nos pecíolos das folhas opostas ao $1^{\circ}$ cacho (Figuras 2 e 3; Tabela 3). Tais decréscimos observados nas concentrações de $\mathrm{N}^{-\mathrm{NO}_{3}}$ na seiva e na matéria seca dos
Tabela 2. Correlações de Pearson entre o peso da matéria seca da parte aérea do tomateiro cultivado em solos de Viçosa (Vi) e de Três Marias (TM), e os teores de $\mathrm{N}^{-\mathrm{NO}_{3}}$ da seiva (S) e da matéria seca do pecíolo (P), e os teores de N-orgânico (N-org) e N-total (N-tot) da matéria seca do limbo das folhas opostas ao $1^{\circ}(\mathrm{C} 1)$ e ao $3^{\circ}(\mathrm{C} 3)$ cachos. Viçosa, Universidade Federal de Viçosa, 1995/1996.

\begin{tabular}{|c|c|c|}
\hline \multirow{3}{*}{$\begin{array}{c}\text { Formas de nitrogênio } \\
\text { analisadas }\end{array}$} & \multicolumn{2}{|c|}{ Matéria seca da parte aérea } \\
\hline & \multicolumn{2}{|c|}{ Coeficiente de correlação linear (r) } \\
\hline & Solo Vi & Solo TM \\
\hline $\mathrm{N}-\mathrm{NO}_{3} \mathrm{~S}(\mathrm{C} 1 ; \mathrm{mg} / \mathrm{L})$ & $0,611 * *$ & $0,672 * *$ \\
\hline $\mathrm{N}-\mathrm{NO}_{3} \mathrm{P}(\mathrm{C} 1 ; \mathrm{mg} / \mathrm{kg})$ & $0,393 *$ & $0,604 * *$ \\
\hline $\mathrm{N}$-org (C1; dag/kg) & $0,287^{\circ}$ & $0,747 * *$ \\
\hline N-tot $(\mathrm{C} 1 ;$ dag/kg) & $0,292^{\circ}$ & $0,738 * *$ \\
\hline $\mathrm{N}-\mathrm{NO}_{3} \mathrm{~S}(\mathrm{C} 3 ; \mathrm{mg} / \mathrm{L})$ & $0,538 * *$ & $0,564 * *$ \\
\hline $\mathrm{N}-\mathrm{NO}_{3} \mathrm{P}(\mathrm{C} 3 ; \mathrm{mg} / \mathrm{kg})$ & $0,478 * *$ & $0,503 * *$ \\
\hline N-org (C3; dag/kg) & $0,334^{\circ}$ & $0,752 * *$ \\
\hline N-tot $(\mathrm{C} 3 ;$ dag/kg) & $0,341^{\circ}$ & $0,749 * *$ \\
\hline
\end{tabular}

**, * $\mathrm{e}^{\circ}=$ significância aos níveis de 1,5 e $10 \%$ de probabilidade, respectivamente.

Tabela 3. Equações de regressão relacionando os teores de $\mathrm{N}_{-} \mathrm{NO}_{3}$ na seiva (S) e na matéria seca do pecíolo $(\mathrm{P})$, e os teores de $\mathrm{N}$-orgânico (N-org) e de $\mathrm{N}$-total (N-tot) na matéria seca do limbo das folhas opostas ao $1^{\circ}(\mathrm{C} 1)$ e ao $3^{\circ}(\mathrm{C} 3)$ cachos do tomateiro, cultivado em amostras de solos de Viçosa (Vi) e de Três Marias (TM), em função das doses de nitrogênio (D). Viçosa, Universidade Federal de Viçosa, 1995/1996.

\begin{tabular}{lc}
\hline \multicolumn{1}{c}{ Equações } & R2 2 \\
\hline \multicolumn{1}{c}{ Solo Vi } & \\
$\mathrm{N}-\mathrm{NO}_{3}(\mathrm{SC} 1)=-2.655,9+719,9^{* *} \mathrm{D}^{0,5}-23,162^{* *} \mathrm{D}$ & 0,358 \\
$\mathrm{~N}-\mathrm{NO}_{3}(\mathrm{PC} 1)=-10.017,8+2.719,97^{* *} \mathrm{D}^{0,5}-99,65^{* *} \mathrm{D}$ & 0,419 \\
$\mathrm{~N}-\mathrm{org}(\mathrm{C} 1)=1,37+0,438^{* *} \mathrm{D}^{0,5}-0,017^{* *} \mathrm{D}$ & 0,437 \\
$\mathrm{~N}-$ tot $(\mathrm{C} 1)=+1,28+0,465^{* *} \mathrm{D}^{0,5}-0,018^{* *} \mathrm{D}$ & 0,394 \\
$\mathrm{~N}-\mathrm{NO}$ \\
3
\end{tabular}

**, * $\mathrm{e}^{\circ}=$ significância aos níveis de 1,5 e $10 \%$ de probabilidade; 


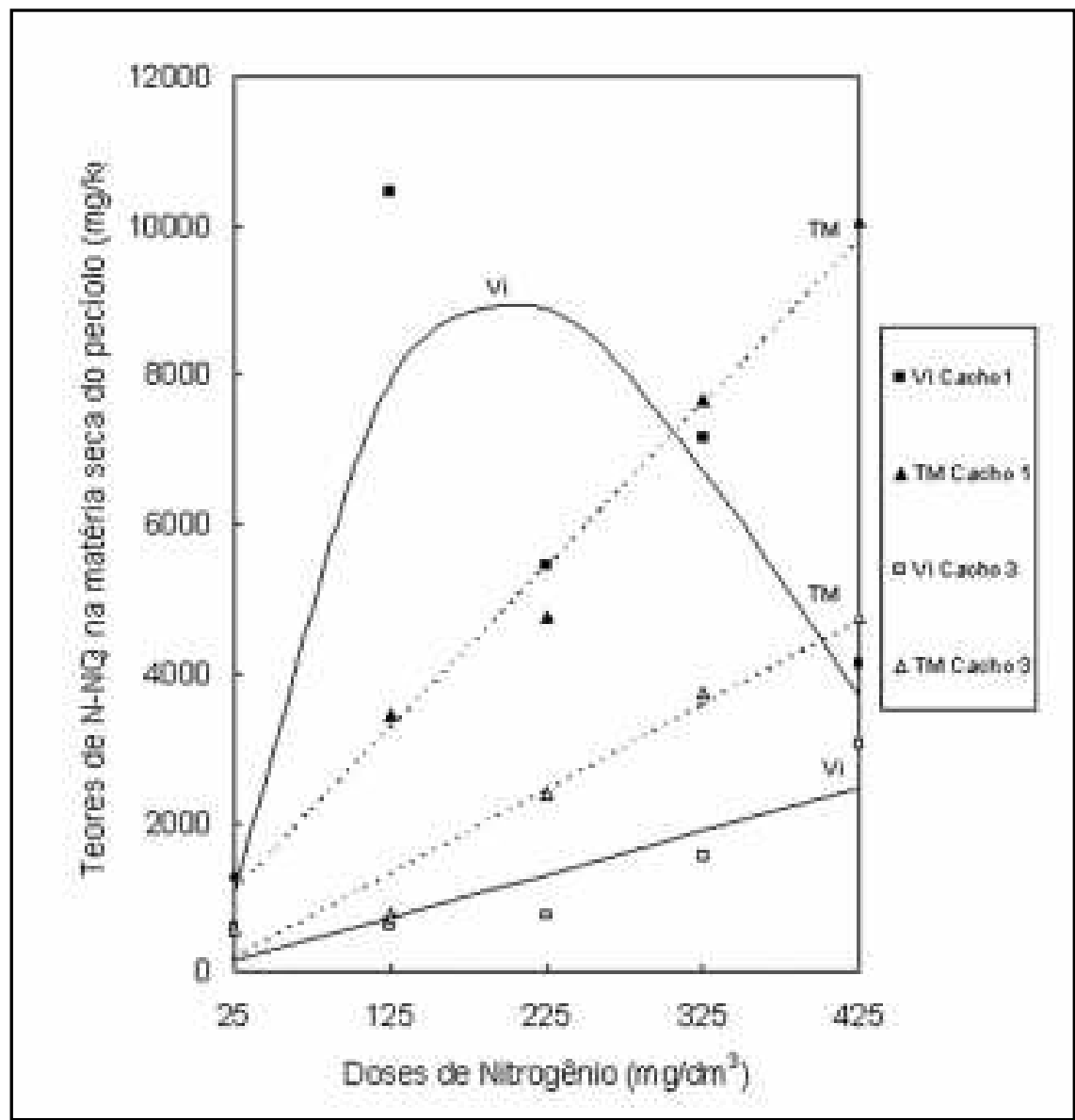

Figura 2. Teores de $\mathrm{N}_{-} \mathrm{NO}_{3}$ na matéria seca dos pecíolos das folhas opostas ao primeiro e ao terceiro cacho do tomateiro, em solos de Viçosa (Vi) e de Três Marias (TM), em função de doses de nitrogênio. Viçosa, Universidade Federal de Viçosa, 1995/1996.

Tabela 4. Equações ajustadas para estimativa dos teores de $\mathrm{N}-\mathrm{NO}_{3}(\mathrm{mg} / \mathrm{kg})$ na matéria seca de pecíolos, e de N-org (dag/kg) e N-tot (dag/kg) na matéria seca do limbo das folhas opostas ao $1^{\circ}(\mathrm{C} 1)$ e ao $3^{\circ}(\mathrm{C} 3)$ cachos de frutos do tomateiro, cultivado em amostras de solos de Viçosa (Vi) e de Três Marias (TM), em função dos teores de $\mathrm{N}^{-\mathrm{NO}_{3}}(\mathrm{mg} / \mathrm{L})$ na seiva dos pecíolos. Viçosa, Universidade Federal de Viçosa, 1995/1996.

\begin{tabular}{|c|c|}
\hline Equações & $\mathbf{R}^{2}$ \\
\hline \multicolumn{2}{|l|}{ Solo Vi } \\
\hline $\mathrm{N}-\mathrm{NO}_{3}(\mathrm{C} 1)=-218,3+2,64 * * \mathrm{X}$ & 0,777 \\
\hline $\mathrm{N}-\operatorname{org}(\mathrm{C} 1)=2,95+0,00037 * * \mathrm{X}$ & 0,564 \\
\hline N-tot $(\mathrm{C} 1)=2,95+0,00041 * \mathrm{X}$ & 0,579 \\
\hline $\mathrm{N}-\mathrm{NO}_{3}(\mathrm{C} 3)=-630,2+2,63 * * X$ & 0,924 \\
\hline N-org $(C 3)=-0,72+0,009 * * X-0,000005 * * X^{2}$ & 0,377 \\
\hline N-tot $(C 3)=-0,0144+0,0088 * * X-0,0000051 * * X^{2}$ & 0,370 \\
\hline \multicolumn{2}{|l|}{ Solo TM } \\
\hline $\mathrm{N}-\mathrm{NO}_{3}(\mathrm{C} 1)=655,2+2,47 * * \mathrm{X}$ & 0,986 \\
\hline $\mathrm{N}-\operatorname{org}(\mathrm{C} 1)=2,91+0,0015^{* *} \mathrm{X}-0,0000002 * * \mathrm{X}^{2}$ & 0,968 \\
\hline $\mathrm{N}-$ tot $(\mathrm{C} 1)=2,92+0,0015^{* *} \mathrm{X}-0,0000002 * * \mathrm{X}^{2}$ & 0,970 \\
\hline $\mathrm{N}-\mathrm{NO}_{3}(\mathrm{C} 3)=-202,7+2,08 * * \mathrm{X}$ & 0,966 \\
\hline $\mathrm{N}-$ org $(\mathrm{C} 3)=2,01+0,0026 * * \mathrm{X}-0,00000063 * * \mathrm{X}^{2}$ & 0,798 \\
\hline N-tot $(C 3)=2,03+0,0026 * * X-0,00000062 * * X^{2}$ & 0,810 \\
\hline
\end{tabular}

** e * = significância aos níveis de 1 e $5 \%$ de probabilidade; pecíolos com o desenvolvimento do tomateiro são relatados por diversos autores (Coltman, 1987; Hochmuth, 1994; Mason \& Wilcox, 1982). Com o desenvolvimento da planta, o aumento da demanda em $\mathrm{N}$ pela parte aérea não é acompanhado, proporcionalmente, pela quantidade de $\mathrm{N}$ absorvida pelo sistema radicular, fazendo com que as concentrações de $\mathrm{NO}_{3}$ nos pecíolos das folhas do tomateiro apresentem um gradiente decrescente da base para o ápice.

As concentrações de $\mathrm{N}$-org e de Ntot no limbo das folhas opostas ao $3^{\circ}$ cacho apresentaram comportamento descritos pelos modelos cúbico no solo Vi e pelo quadrático no solo TM (Figuras 4 e 5; Tabela 3). O N-tot presente no limbo das folhas amostradas constituiuse quase que totalmente por N-orgânico, sendo muito pequena a contribuição do $\mathrm{N}_{-} \mathrm{NO}_{3}$ (Figuras 4 e 5).

A relação entre os valores de $\mathrm{N}-\mathrm{NO}_{3}$ na matéria seca dos pecíolos e os valores de $\mathrm{N}-\mathrm{NO}_{3}$ na seiva destes foram lineares nos dois solos e nas duas amostragens. Somente o modelo ajustado na $1^{\text {a }}$ amostragem para o solo $\mathrm{Vi}$ apresentou $\mathrm{R}^{2}$ próximo de 0,78 ; todos os demais, apresentaram valores de $\mathrm{R}^{2}$ superiores a 0,92 (Tabela 4). Em estudos semelhantes realizados na Califórnia, com tomateiro cultivado no campo, Hartz et al.(1993) obtiveram valores de $R^{2}$ igual a 0,83 na estimativa desta relação.

A relação entre os valores de $\mathrm{N}$-org e de N-tot na matéria seca do limbo, e os valores de $\mathrm{N}_{-} \mathrm{NO}_{3}$ na seiva do pecíolo foram semelhantes entre si, com valores de $\mathrm{R}^{2}$ muito próximos, em ambas as amostragens, em cada solo (Tabela 4). Entretanto, os valores de $\mathrm{R}^{2}$ das equações obtidas para o solo TM foram sempre maiores que aqueles das equações obtidas para o solo Vi, indicando que a capacidade preditiva foi melhor para os resultados obtidos neste solo (Tabela 4).

Os níveis críticos das formas de $\mathrm{N}$ nas partes analisadas das plantas foram sempre maiores no solo TM que no solo Vi (Tabela 5). Tal fato, associado à menor produção de matéria seca de frutos e da parte aérea das plantas cultivadas no solo TM, indicam que houve algum impedimento ao crescimento das plantas cultivadas neste solo. Originalmen- 
te, o solo TM é pobre em $\mathrm{P}, \mathrm{K}, \mathrm{Ca}$ e $\mathrm{Mg}$, e possui baixa capacidade de retenção de água (Tabela 1). Levando-se em conta que não foi aplicado micronutrientes ao solo no plantio (somente em cobertura), e que o experimento foi conduzido em casa-de-vegetação no verão, é provável que, a carência de algum micronutriente (talvez $\mathrm{Zn}$ e, ou, B) associada à menor disponibilidade de água tenham exercido efeito depressivo sobre o crescimento das plantas, causando acúmulo de $\mathrm{N}$ nestas plantas. Em cada um dos solos, os níveis críticos das formas de $\mathrm{N}$ nas partes analisadas das plantas foram maiores nas folhas opostas ao $1^{\circ}$ cacho que naquelas opostas ao $3^{\circ}$ (Tabela 5), tendo a provável explicação já sido relatada anteriormente.

As concentrações de $\mathrm{N}-\mathrm{NO}_{3}$ na seiva dos pecíolos obtidas no presente trabalho encontram-se acima da faixa de suficiência de $\mathrm{N}-\mathrm{NO}_{3}$ na seiva da folha recentemente madura de tomateiro cultivado em estufa, proposta por Hochmuth (1994), os quais são 10001200 e $800-1000 \mathrm{mg} / \mathrm{L}$ de $\mathrm{N}_{-} \mathrm{NO}_{3}$ na seiva, para as fases que vão do transplantio até o $2^{\circ}$ cacho e deste ao $5^{\circ}$ cacho, respectivamente. Esses valores foram observados em plantas cultivadas no campo, e foram obtidos por meio de testes colorimétricos, ao contrário do presente estudo, no qual as plantas foram cultivadas em vasos e as concentrações de $\mathrm{N}_{-} \mathrm{NO}_{3}$ na seiva foram determinadas por método potenciométrico.

Os resultados relativos às concentrações de $\mathrm{N}_{-} \mathrm{NO}_{3}$ na seiva do pecíolo para o tomateiro cultivado no solo Vi são similares aqueles propostos por Coltman (1987) para plantas cultivadas em vasos contendo $13 \mathrm{dm}^{3}$ de solo, e que apresentaram a máxima produção de frutos comercializáveis associada à concentrações de $\mathrm{N}_{-} \mathrm{NO}_{3}$ na seiva de 2138 e 1091 $\mathrm{mg} / \mathrm{L}$, para o início do florescimento e para o início da frutificação, respectivamente.

Os níveis críticos de $\mathrm{N}-\mathrm{NO}_{3}$ na matéria seca dos pecíolos das plantas cultivadas no solo Vi e no solo TM, na amostragem realizada por ocasião do aparecimento do $3^{\circ}$ cacho (Tabela 5), encontram-se abaixo da faixa de suficiência proposta por Geraldson \& Tyler (1990). Esses autores propõe como deficientes, os teores de $\mathrm{N}-\mathrm{NO}_{3}$ na matéria

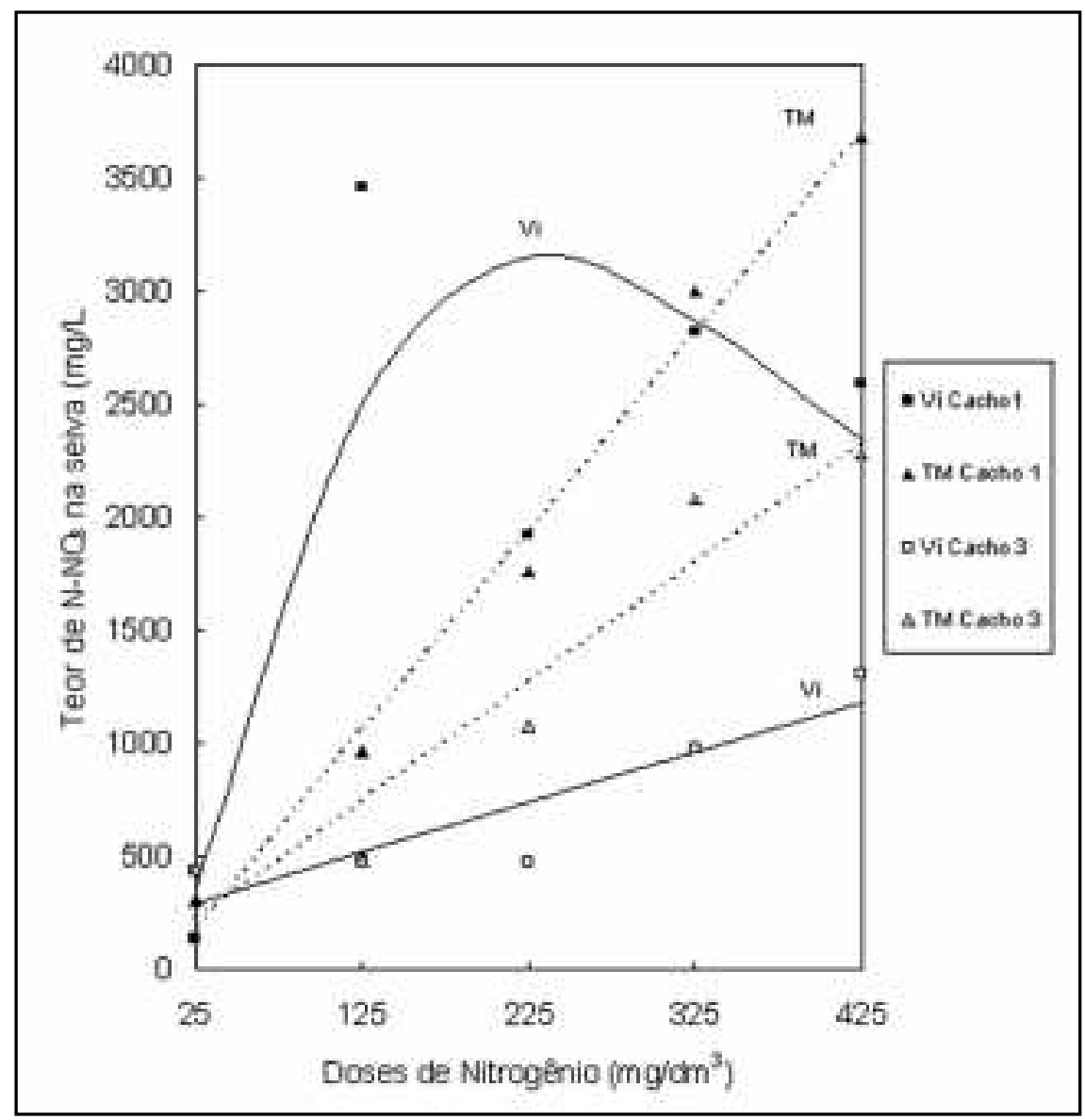

Figura 3. Teores de $\mathrm{N}^{-\mathrm{NO}_{3}}$ na seiva dos pecíolos das folhas opostas ao primeiro e ao terceiro cacho do tomateiro, em solos de Viçosa (Vi) e de Três Marias (TM), em função de doses de nitrogênio. Viçosa, Universidade Federal de Viçosa, 1995/1996.

Tabela 5. Níveis críticos de $\mathrm{N}_{-} \mathrm{NO}_{3}$ na seiva e na matéria seca do pecíolo, e de $\mathrm{N}$-org e $\mathrm{N}$-tot na matéria seca do limbo das folhas opostas ao $1^{\circ} \mathrm{e}$ ao $3^{\circ}$ cachos de tomateiro cultivado em amostras de solos de Viçosa (Vi) e Três Marias (TM). Viçosa, Universidade Federal de Viçosa, 1995/1996.

\begin{tabular}{lcccc}
\hline \multirow{2}{*}{ Formas de nitrogênio analis adas } & \multicolumn{2}{c}{ Vi } & \multicolumn{2}{c}{ TM } \\
\cline { 2 - 5 } & $\mathbf{1}^{\mathbf{0}}$ cacho & $\mathbf{3}^{\mathbf{0}}$ cacho & $\mathbf{1}^{\mathbf{0}}$ cacho & $\mathbf{3}^{\mathbf{0}}$ cacho \\
\hline $\mathrm{N}-\mathrm{NO}_{3}$ na seiva do pecíolo $(\mathrm{mg} / \mathrm{L})$ & 2581 & 1085 & 2616 & 1690 \\
$\mathrm{~N}^{-} \mathrm{NO}_{3}$ na matéria seca do pecíolo $(\mathrm{mg} / \mathrm{kg})$ & 5017 & 2242 & 7101 & 3312 \\
N-org na matéria seca do limbo $(\mathrm{dag} / \mathrm{kg})$ & 3,43 & 3,43 & 5,43 & 4,42 \\
N-tot na matéria seca do limbo $(\mathrm{dag} / \mathrm{kg})$ & 3,48 & 3,49 & 5,75 & 4,44 \\
\hline
\end{tabular}

seca dos pecíolos da $4^{\text {a }}$ folha à partir do ápice, extraídos com ácido acético, de $8000 \mathrm{mg} / \mathrm{kg}$ no início do florescimento e de $6000 \mathrm{mg} / \mathrm{kg}$ no estádio de frutos pequenos (2,5 cm de diâmetro), para tomateiro cultivado no campo para fins industriais. As concentrações de $\mathrm{N}-\mathrm{NO}_{3}$ na matéria seca do pecíolo das folhas opostas ao $1^{\circ}$ cacho encontram-se abaixo do valor associado à máxima produção de matéria seca pelo tomateiro em condi- ções de solução nutritiva obtido por Fontes et al. (1995), para plantas amostradas no início de florescimento e utilizando todos os pecíolos da planta.

Os resultados obtidos mostram que a utilização do microeletrodo representa uma alternativa viável no diagnóstico da nutrição nitrogenada do tomateiro, devido a rapidez e facilidade de uso, e à relação existente com as formas de $\mathrm{N}$ usualmente determinadas em laboratório. 


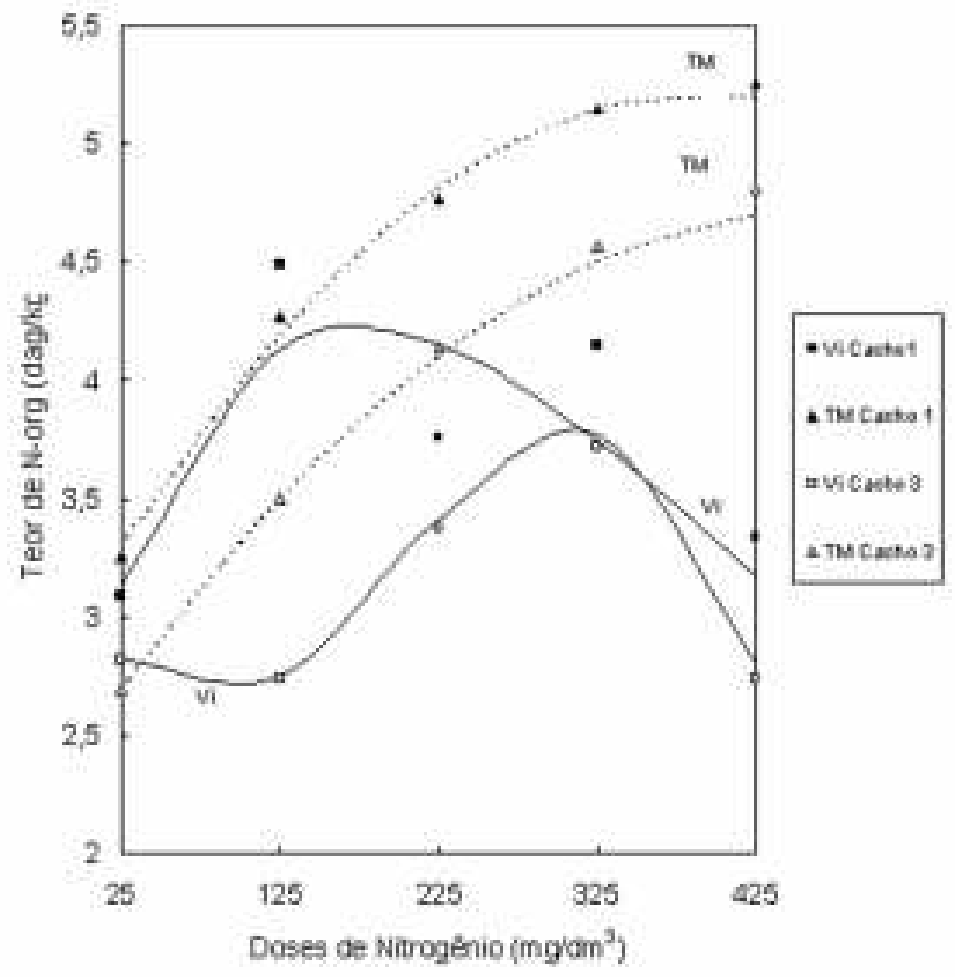

Figura 4. Teores de N-orgânico no limbo das folhas opostas ao primeiro cacho e ao terceiro cacho do tomateiro, em solos de Viçosa (Vi) e de Três Marias (TM), em função de doses de nitrogênio. Viçosa, Universidade Federal de Viçosa, 1995/1996.

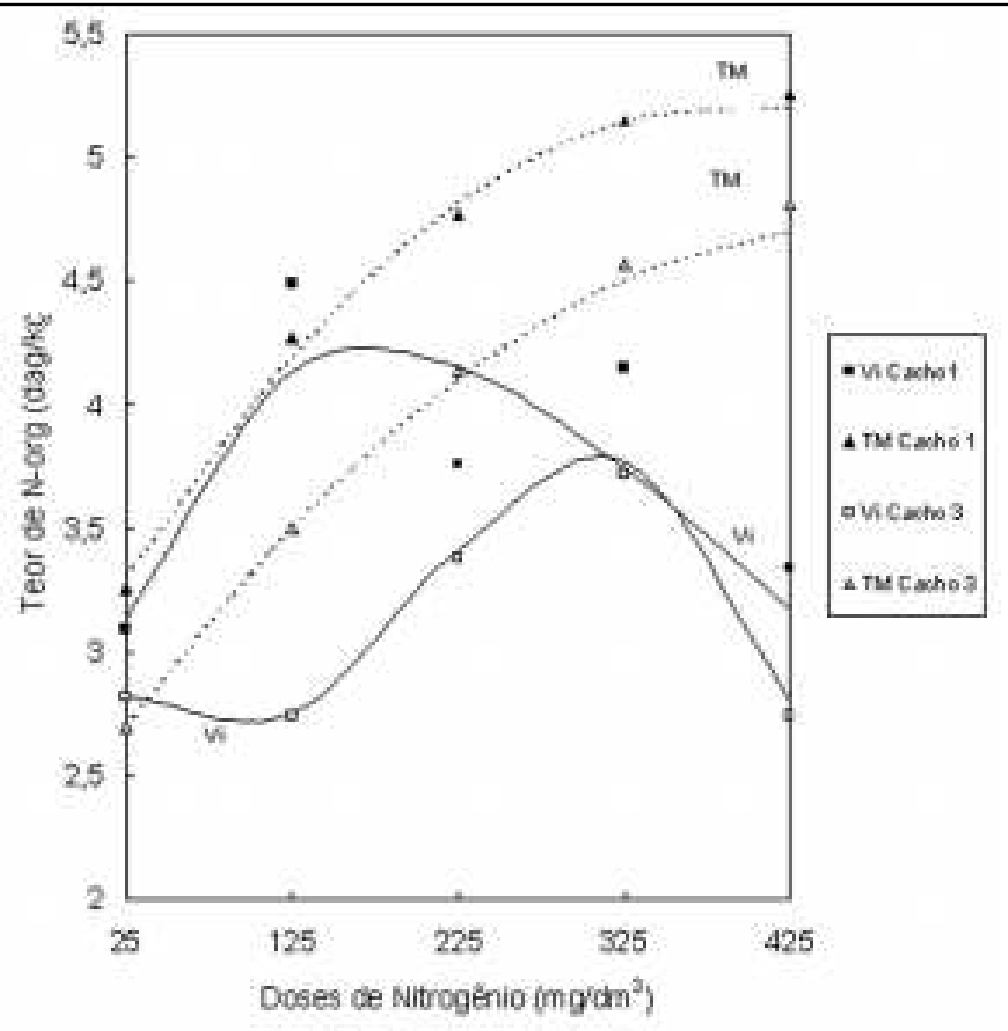

Figura 5. Teores de N-total no limbo das folhas opostas ao primeiro cacho e ao terceiro cacho do tomateiro, em solos de Viçosa (Vi) e de Três Marias (TM), em função de doses de nitrogênio. Viçosa, Universidade Federal de Viçosa, 1995/1996.

\section{LITERATURA CITADA}

BINFORD, G.D.; BLACKMER, A.M.; CERRATO, M.E. Relationships between corn yields and soil nitrate in late spring. Agronomy Journal, v. 84, p. 53-59, 1992.

BLACKMER, A.M.; MORRIS, T.F.; MEESE, B.G.; MALLARINO, A.P. Soil testing to optimize nitrogen management for corn. Leopold Center, Iowa State University of Science. Pm-1521, 1993. 4 p.

CATALDO, D.A.; HAROON, M.; SCHRADER, L.E.; YOUNES, V.L. Rapid colorimetric determination of nitrate in plant tissue by nitration of salicilic acid. Communications in Soil Science and Plant Analysis, v. 6, p. 71-80, 1975.

COLTMAN, R.R. Sampling considerations for nitrate quick tests of greenhouse-grown tomatoes. Journal of the American Society of Horticultural Science, v. 112, n. 6, p. $922-$ 927, 1987.

COLTMAN, R.R. Yields of greenhouse tomatoes managed to maintain specific petiole sap nitrate levels. Hortscience, v. 23, n. 1, p. 148$151,1988$.

FONTES, P.C.R; GOMES, J.M.; PEREIRA, P.R.G.; MARTINEZ, H.E.P. Nível crítico de $\mathrm{N}-\mathrm{NO}_{3}$ em pecíolos de tomateiro extraído por diferentes métodos. Horticultura Brasileira, Brasília, v. 13, n. 1, p. 11-13, 1995.

GERALDSON, C.M.; TYLER, K.B. Plant analysis as an aid in fertilizing vegetable crops. In: WESTERMAN, R.L., ed. Soil Testing and Plant Analysis. $3^{\mathrm{a}}$ ed. Madison. Soil Science Society of America. 1990. p. 549-562.

HARTZ, T.K.; SMITH, R.F.; LeSTRANGE, M.; SCHULBACH, K.F. On-farm monitoring of soil and crop nitrogen status by nitrateselective electrode. Communications in Soil Science and Plant Analysis, v. 24, n. 19/20, p. 2607-2615, 1993.

HOCHMUTH, G.J. Efficiency ranges for nitratenitrogen and potassium for vegetable petiole quick sap tests. HortTechnology, v. 4, p. 218222, 1994.

JACKSON, M.L. Analisis Químico de Suelos. Ediciones Omega, S.A. Barcelona, 1982, $662 \mathrm{p}$.

MACKERRON, D.K.L.; YOUNG, M.W.; DAVIES, H.V. A method to optimize N application in relation to soil supply of $\mathrm{N}$, and yield of potato. Plant and Soil, v. 154, p. 139-144, 1993.

MASON, S.C.; WILCOX, G.E. Nitrogen status evaluation of tomato plants. Journal of the American Society of Horticultural Science, v. 107, n. 3, p. 483-486, 1982.

MINNOTI, P.L.; HANKINSON, T.J.; GRUBINGERE, V.P.; WIEN, H.C. Whole leaves versus petioles for assessing the nitrogen "status" of tomatoes. Hortscience, v. 24, n. 1, p. 84-86, 1989.

NEETESON, J.J. Evaluation of the performance of three advisory methods for nitrogen fertilization of sugar beet and potatoes. Netherlands Journal of Agricultural Science, v. 37, p. 143-155, 1989. 
NEETESON, J.J.; GREENWOOD, D.J.; HABETS, J.M.H. Dependence of soil mineral $\mathrm{N}$ on fertilizer application. Plant and Soil, v. 91, p. 417-420, 1986

NEETESON, J.J.; ZWETSLOOT, H.J.C. An analysis of the response of sugar beet and potatoes to fertilizer nitrogen and soil mineral nitrogen. Netherlands Journal of Agricultural Science, v. 37, p. 129-141, 1989.
SARRO, M.J.; CADAHIA, C.; CARPENA O. Balance ionico en savia como indice de nutricion del tomate. Nueva metodologia analitica aplicable "in situ". Anales de Edafologia e Agrobiologia, v. 44, n. 5/6, p. 799-812, 1985.
SARRO, M.J.; CADAHIA, C.; CARPENA O. Evolutionary nutrient balances as indexes in the diagnosis of nutrition in tomato cultivation (Lycopersicon esculentum Mill), cvs. Marglobe and Super-Roma. Agrochimica, v. 31, n. 1/2, p. 54-64, 1987.

SILVA, J.B.C.; NAKAGAWA, J. Confecção e avaliação de péletes de sementes de alface. Horticultura Brasileira, Brasília, v. 16 , n. 2 , p. 151 - 158 , novembro 1998.

\title{
Confecção e avaliação de péletes de sementes de alface. ${ }^{1}$
}

\author{
João Bosco C. da Silva ${ }^{2}$; João Nakagawa ${ }^{3}$ \\ ${ }^{2}$ Embrapa Hortaliças, C. Postal 218, CEP 70.359-970 Brasília - DF; ${ }^{3}$ UNESP - Faculdade de Ciências Agronômicas. C. Postal 237, CEP \\ 18.603-970 Botucatu - SP.
}

\section{RESUMO}

Para este trabalho, os péletes foram confeccionados em betoneiras, onde as sementes rolam umas sobre as outras, recebendo aos poucos e alternadamente os ingredientes de enchimento e cimentante, até atingirem o tamanho desejado, sendo então secados e classificados. Foram confeccionados péletes de semente de alface com areia fina, areia grossa, calcário fino e calcário grosso, utilizando como cimentante, bentonita diluída em água a 7,5\% ou cola à base de acetato de polivinila (PVA) a 30\%. Parte dos péletes recebeu acabamento com calcário calcítico mais cola à base de PVA. Os péletes pequenos, confeccionados com areia fina ou com a mistura de areia e calcário, com ou sem acabamento, apresentaram os melhores resultados, não diferindo da germinação e da velocidade de crescimento de plântulas obtidas de sementes nuas. A utilização de calcário prejudicou o crescimento das plântulas e o prejuízo foi mais acentuado para os péletes grandes. Os péletes confeccionados com calcário e cola à base de PVA foram os mais resistentes ao esmagamento e os péletes confeccionados com areia, utilizando bentonita como cimentante, os de menor resistência. A cola à base de PVA atuou como cimentante mais forte que a bentonita e a aplicação da camada de acabamento com cola à base de PVA aumentou a firmeza dos péletes. Os péletes confeccionados com areia apresentaram maior velocidade de dissolução, mas todos os péletes testados se dissolveram em menos de um minuto. Todos eles apresentaram baixo grau de umidade (média de $0,45 \%$ ) e baixa higroscopicidade (cerca de $0,6 \%$ ).

Palavras-chave: Lactuca sativa, peletização, higroscopicidade, resistência.

\begin{abstract}
Production and testing of lettuce seed pellets.

In this work, pellets were produced in a small concrete mixerlike machine (pan), where seeds rolled over one another, receiving gradually and alternately coating (stuffing) and cement (adhesive) ingredients, until they reached the required size. Seeds were then dried and classiffied. Lettuce seeds were pelleted with fine sand, coarse sand, fine lime or coarse lime, using bentonite diluted in water at $7,5 \%$, or polivinil acetate glue (PVA) at $30 \%$, as cement. A portion of these pellets were covered with a thin layer of lime plus PVA. The small pellets made of fine sand plus lime with or without the fine finish cover presented the best results. Rates of germination and seedling development were similar to those obtained with bare seeds. Lime usage delayed seedling growth, being more pronounced on larger pellets. Using lime plus PVA glue resulted in the most physical resistance, whereas the use of sand plus bentonite, resulted in the least resistance; fine finish enhanced the pellet resistance. Pellets with PVA glue were more resistant than those with bentonite. Coats of sand were most quickly dissolved, although pellets tested dissolved in less than one minute. The water content in each type of pellet was less than $0.45 \%$, with low higroscopicity (less than $0.6 \%$ ).
\end{abstract}

Keywords: Lactuca sativa, coat, pelleting, resistance, higroscopicity.

\section{(Aceito para publicação em 28 de agosto de 1998)}

$\mathrm{O}$ péletes de semente são confeccionados basicamente com um material seco, não solúvel, inerte e de granulometria fina, denominado de enchimento, e um cimentante que deve ser um adesivo não fitotóxico, solúvel em água e de reidratação rápida.
A camada de peletização é relativamente delgada (menor que $2 \mathrm{~mm}$ de espessura) mas é constituída por partículas finas, bem arranjadas e aderidas entre si, formando uma capa intimamente aderida à superfície da semente. Esta constituição implica em se ter o míni- mo de porosidade e o máximo de força de retenção da água, difícultando a sua drenagem e, conseqüentemente, restringindo a troca gasosa entre a semente e o ambiente externo ao pélete, causando o retardamento na germinação (Sachs $e t$ al., 1982; Tonkin, 1984). 